\title{
Evenly spaced data points and radial basis functions
}

\author{
L. T. Luh \\ Department of Mathematics, Providence University, Taiwan
}

\begin{abstract}
The purpose of this article is to introduce a kind of data setting to handle radial basis functions. Traditionally the meshless method RBF uses scattered data setting to do interpolations. This approach faces two hard problems. First, the optimal choice of the shape parameters contained in smooth radial functions are not easy to find. Second, the crucial constant $\omega$ in the exponential-type error bound, which is $O\left(\omega^{\frac{1}{d}}\right)$, is too large, making this error bound meaningful only when the fill distance $d$ is extremely small. However, in the evenly spaced data setting, an error bound of the form $O\left(\sqrt{d} \omega^{\frac{1}{d}}\right)$ is established where $\omega$ is much sharper than that of the former one. What's important is that whenever this error bound is adopted, the optimal choice of the shape parameter can always be found with the fill distance $d$ of reasonable size.

We express the effect of the shape parameter $c$ by explicitly defined functions and present concrete criteria of the optimal choice of $c$, which do not require too many data points.
\end{abstract}

Keywords: radial basis function, shifted surface spline, shape parameter, interpolation.

\section{Introduction}

This article is intended only to open a window for the topic of choosing the shape parameter optimally. We try to avoid mentioning complicated theory and heavy techniques. Rather, we present concrete criteria of choosing the shape parameter.

The smooth RBFs, multiquadric, inverse multiquadric, gaussian, and shifted surface spline, all contain a shape parameter, usually denoted by $c$. Recently Cheng made a comprehensive study of its choice for MQ, IMQ and GA in Cheng [1], while the choice of $c$ in the shifted surface spline was missing. We therefore focus 
on this kind of RBF whose theoretical properties are equally complicated. This function is defined as follows.

$$
\begin{aligned}
h(x):= & (-1)^{m}\left(|x|^{2}+c^{2}\right)^{\frac{\lambda}{2}} \log \left(|x|^{2}+c^{2}\right)^{\frac{1}{2}}, \lambda \in Z_{+}, m=1+\frac{\lambda}{2}, c>0, \\
& x \in R^{n}, \lambda, n \text { even, }
\end{aligned}
$$

where $|x|$ is the Euclidean norm of $x, \log$ denotes the natural logarithm, and $\lambda, c$ are constants. The constant $c$ is called shape parameter whose optimal choice is a big problem. In order to provide a simple and concrete insight into our approach, we will deal with the case $\lambda=2$ only.

Then, for any scattered set of data points $\left(x_{1}, f\left(x_{1}\right)\right), \ldots,\left(x_{N}, f\left(x_{N}\right)\right)$, there is a unique function

$$
s(x):=\sum_{j=1}^{N} c_{j} h\left(x-x_{j}\right)+p(x)
$$

interpolating these data points, where $c_{1}, \ldots, c_{N}$ are constants to be determined and $p(x)$ is a polynomial of degree $\leq m-1$. The only requirement for the data points is that $x_{1}, \ldots, x_{N}$ should be polynomially nondegenerate.

The interpolated functions belong to two function spaces.

Definition 1.1. For any $\sigma>0$, the class of band-limited functions $f$ in $L^{2}\left(R^{n}\right)$ is defined by

$$
B_{\sigma}:=\left\{f \in L^{2}\left(R^{n}\right): \hat{f}(\xi)=0 \text { if }|\xi|>\sigma\right\},
$$

where $\hat{f}$ denotes the Fourier transform of $f$.

A larger function space is defined as follows.

Definition 1.2. For any $\sigma>0$, the class of educated functions is

$$
E_{\sigma}:=\left\{f \in L^{2}\left(R^{n}\right): \int|\hat{f}(\xi)|^{2} e^{\frac{|\xi|^{2}}{\sigma}} d \xi<\infty\right\},
$$

where $\hat{f}$ denotes the Fourier transform of $f$. For each $f \in E_{\sigma}$, its norm is

$$
\|f\|_{E_{\sigma}}:=\left\{\int|\hat{f}(\xi)|^{2} e^{\frac{|\xi|^{2}}{\sigma}} d \xi\right\}^{\frac{1}{2}} .
$$

Although we restrict our interpolated functions to the two classes, other functions can be handled by triangle inequality. For example, as pointed out in Wendland [2], any function in the Sobolev space can be interpolated well by a band-limited function. Then we use $s(x)$ to interpolate that band-limited function at the same data points.

Different from Cheng's approach, we require that the data points be evenly spaced in the following sense. 
Let $E$ be an n-dimensional simplex in $R^{n}$ with vertices $v_{1}, \ldots, v_{n+1}$. For any point $x \in E$, its barycentric coordinates are the numbers $\lambda_{1}, \ldots, \lambda_{n+1}$ satisfying

$$
x=\sum_{i=1}^{n+1} \lambda_{i} v_{i}, \quad \sum_{i=1}^{n+1} \lambda_{i}=1, \quad \lambda_{i} \geq 0 \text { for all } i .
$$

The definition of simplex can be found in Fleming [3].

For any $n$-dimensional simplex, the evenly spaced points of degree $\mathrm{k}$ are the points whose barycentric coordinates are of the form

$\left(k_{1} / k, k_{2} / k, \ldots, k_{n+1} / k\right), k_{i}$ nonnegative integers and $k_{1}+\cdots, k_{n+1}=k$.

The interpolation occurs in a simplex and the centers(interpolation points) are evenly spaced points of that simplex. A simplex is just a line segment, triangle and tetrahedron in dimensions 1,2 , and 3 , respectively.

\section{The optimal choice of $c$}

Before introducing our criteria we need some basic definitions.

Definition 2.1. The constant $\rho$ is defined as follows.

(a) Suppose $n>5$. Let $s=\left\lceil\frac{n-5}{2}\right\rceil$. Then

$$
\rho=1+\frac{s}{7}
$$

(b) Suppose $n \leq 5$. Then $\rho=1$.

For any $b_{0}>0$, let $\delta_{0}=\frac{b_{0}}{6}$. Then for any $0<\delta \leq \delta_{0}$, let $c_{0}=72 \rho \delta$ and $c_{1}=12 \rho b_{0}$.

Obviously $c_{0} \leq c_{1}$. This article explores the optimal choice of $c$ in $\left[c_{0}, \infty\right)$.

Based on the complicated theory developed in Luh [4-6], we know that there is a function called $\mathrm{MN}$ function which forms the essential part of the upper bound of $|f(x)-s(x)|$. It's defined by

$$
M N(c)= \begin{cases}\sqrt{8 \rho} c^{\frac{1-n}{4}} e^{c\left[\frac{\sigma}{2}+\frac{\ln \frac{2}{3}}{24 \rho \delta}\right]} & \text { if } c_{0} \leq c \leq c_{1} \\ \sqrt{\frac{2}{3 b_{0}}} c^{\frac{3-n}{4}} e^{\frac{c \sigma}{2}}\left(\frac{2}{3}\right)^{\frac{b_{0}}{2 \delta}} & \text { if } c_{1} \leq c<\infty\end{cases}
$$

for $f \in B_{\sigma}$, and

$$
M N(c)= \begin{cases}\sqrt{8 \rho} c^{\frac{1-n}{4}} \sup _{\xi \in R^{n}}\left\{|\xi|^{\frac{3+n}{4}} e^{\frac{c|\xi|}{2}-\frac{|\xi|^{2}}{2 \sigma}}\right\}\left(\frac{2}{3}\right)^{\frac{c}{24 \rho \delta}} & \text { if } c_{0} \leq c \leq c_{1} \\ \sqrt{\frac{2}{3 b_{0}}} c^{\frac{3-n}{4}} \sup _{\xi \in R^{n}}\left\{|\xi|^{\frac{3+n}{4}} e^{\frac{c|\xi|}{2}-\frac{|\xi|^{2}}{2 \sigma}}\right\}\left(\frac{2}{3}\right)^{\frac{b_{0}}{2 \delta}} & \text { if } c_{1} \leq c<\infty\end{cases}
$$

for $f \in E_{\sigma}$. 
The optimal choice of the shape parameter is then the value minimizing $M N(c)$.

Theorem 2.3 of Luh [6] tells us that for any $b_{0}>0$ and simplex $Q_{0}$ of diameter $b_{0}$, there is a good bound for $|f(x)-s(x)|$ with domain a simplex $Q \subseteq Q_{0}$ of diameter $r \leq b_{0}$. The map $s(x)$ interpolates $f(x)$ at $x_{1}, \cdots, x_{N}$ which are evenly spaced points of degree $k-1$ in $Q$ satisfying $k=\frac{r}{\delta}$ where $\delta \leq \delta_{0}$ can be any positive number.

We divide the criteria into two classes.

\subsection{Band-limited functions}

For $f \in B_{\sigma}$, we have the following cases, where $T:=\frac{\sigma}{2}+\frac{\ln \frac{2}{3}}{24 \rho \delta}$.

Case1. $n=1$ and $T \geq 0$ For any $b_{0}>0$ and positive $\delta<\frac{b_{0}}{6}$, if $n=1$ and $T \geq 0$, the optimal choice of $c$ for $c \in\left[c_{0}, \infty\right)$ is to let $c=c_{0}:=72 \delta$.

Reason: In this case $M N(c)$ in eqn.(3) is increasing on $\left[c_{0}, \infty\right)$.

\section{Numerical Example:}

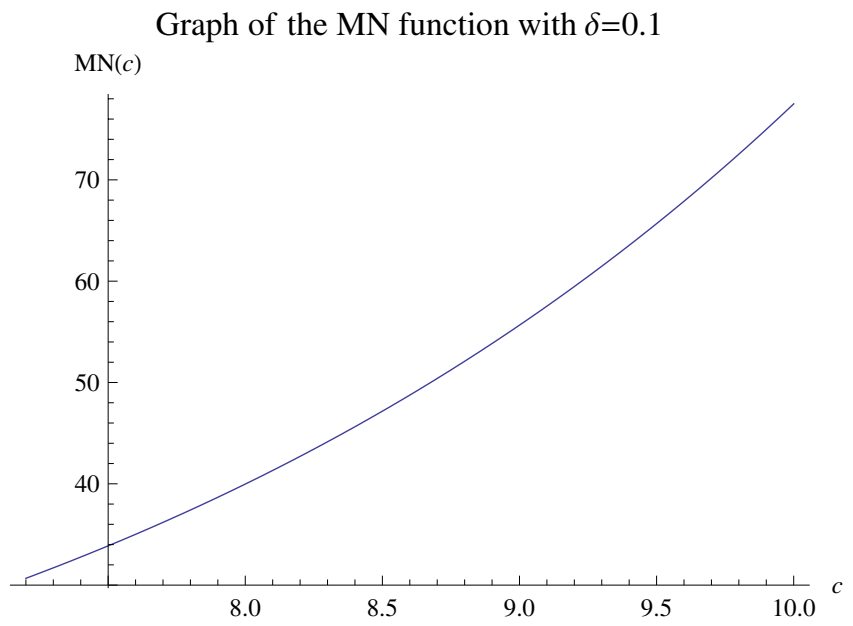

Figure 1: Here $n=1, \sigma=1$ and $b_{0}=1$.

Case2. $n=1$ and $T<0$ For any $b_{0}>0$ and positive $\delta<\frac{b_{0}}{6}$, if $n=1$ and $T<0$, the optimal choice of $c$ for $c \in\left[c_{0}, \infty\right)$ is $c^{*} \in\left[c_{0}, c_{1}\right]$ which minimizes $M N(c)$ of eqn.(3) on $\left[c_{0}, c_{1}\right]$.

Reason: In this case $M N(c)$ in eqn.(3) is increasing on $\left[c_{1}, \infty\right)$. 


\section{Numerical Example:}

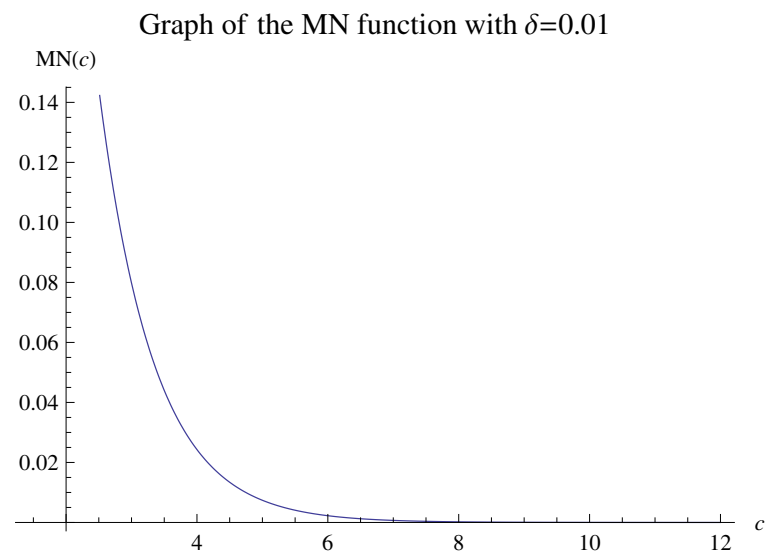

Figure 2: Here $n=1, \sigma=1$ and $b_{0}=1$.

Case3. $n>1$ and $T<0$ For any $b_{0}>0$ and positive $\delta<\frac{b_{0}}{6}$, if $n>1$ and $T<0$, the optimal choice of $c \in\left[c_{0}, \infty\right)$ is the value $c^{*} \in\left[c_{1}, \infty\right)$ which minimizes $M N(c)$ in eqn.(3) on $\left[c_{1}, \infty\right)$.

Reason: In this case $M N(c)$ in eqn.(3) decreases on $\left[c_{0}, c_{1}\right]$.

Remark: In Case3 if $n \leq 3, M N(c)$ will be increasing on $\left[c_{1}, \infty\right)$ and $c^{*}=c_{1}$.

\section{Numerical Example:}

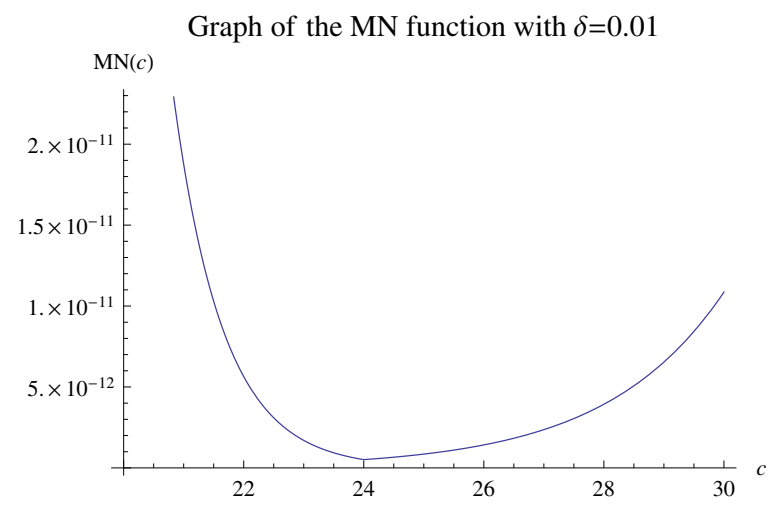

Figure 3: Here $n=2, \sigma=1$ and $b_{0}=2$. 
Now we introduce the last case of band-limited functions.

Case4. $n>1$ and $T \geq 0$ For any $b_{0}>0$ and positive $\delta<\frac{b_{0}}{6}$, if $n>1$ and $T \geq 0$, the optimal choice of $c \in\left[c_{0}, \infty\right)$ is either $c^{*} \in\left[c_{0}, c_{1}\right]$ or $c^{* *} \in\left[c_{1}, \infty\right)$, depending on $M N\left(c^{*}\right) \leq M N\left(c^{* *}\right)$ or $M N\left(c^{* *}\right) \leq M N\left(c^{*}\right)$, where $c^{*}$ and $c^{* *}$ minimize $M N(c)$ in eqn.(3) on $\left[c_{0}, c_{1}\right]$ and $\left[c_{1}, \infty\right)$, respectively.

Reason: In this case $M N(c)$ in eqn.(3) may not be monotonic on both $\left[c_{0}, c_{1}\right]$ and $\left[c_{1}, \infty\right)$. However, if $n \leq 3, M N(c)$ will be increasing on $\left[c_{1}, \infty\right)$ and $c^{* *}=c_{1}$.

\section{Numerical Example:}

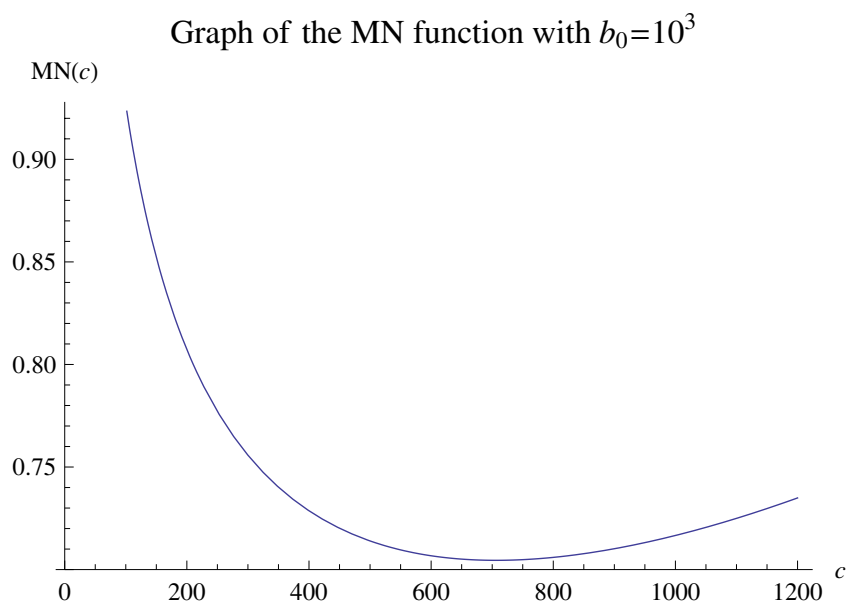

Figure 4: Here $n=2, \sigma=1.127$ and $\delta=0.03$.

\subsection{Educated functions}

We now deal with functions in $E_{\sigma}$.

Case1. $n \leq 3$ For any $b_{0}>0$ and positive $\delta<\frac{b_{0}}{6}$, if $n \leq 3$, the optimal choice of $c \in\left[c_{0}, \infty\right)$ is $c^{*} \in\left[c_{0}, c_{1}\right]$ which minimizes $M N(c)$ in eqn.(4) on $\left[c_{0}, c_{1}\right]$.

Reason: In this case $M N(c)$ is increasing on $\left[c_{1}, \infty\right)$. Hence its minimum value happens in $\left[c_{0}, c_{1}\right]$. 


\section{Numerical Example:}

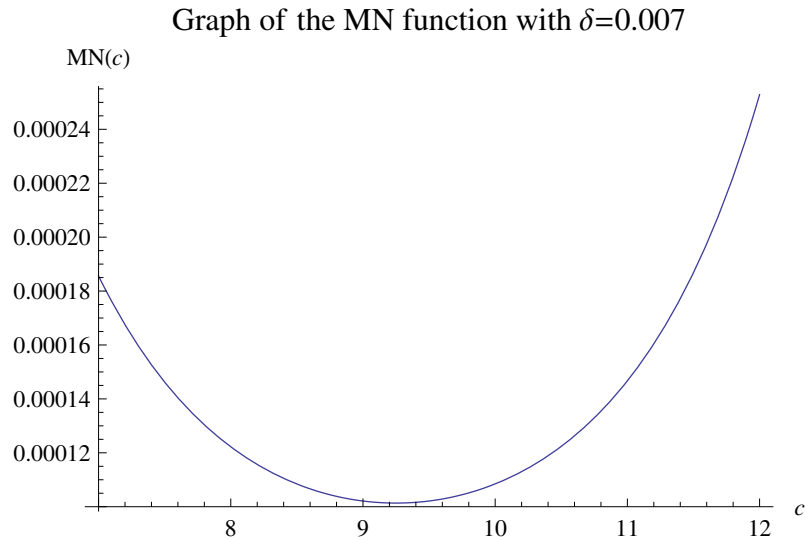

Figure 5: Here $n=2, \sigma=1$ and $b_{0}=1$.

Case2. $n>3$ For any $b_{0}>0$ and positive $\delta<\frac{b_{0}}{6}$, if $n>3$, the optimal choice of $c \in\left[c_{0}, \infty\right)$ is either $c^{*} \in\left[c_{0}, c_{1}\right]$ or $c^{* *} \in\left[c_{1}, \infty\right)$, depending on $M N\left(c^{*}\right) \leq M N\left(c^{* *}\right)$ or $M N\left(c^{* *}\right) \leq M N\left(c^{*}\right)$, where $c^{*}$ and $c^{* *}$ minimize $M N(c)$ in eqn.(4) on $\left[c_{0}, c_{1}\right]$ and $\left[c_{1}, \infty\right)$, respectively.

Reason: In this case $M N(c)$ may not be monotonic on both $\left[c_{0}, c_{1}\right]$ and $\left[c_{1}, \infty\right)$.

\section{Numerical Example:}

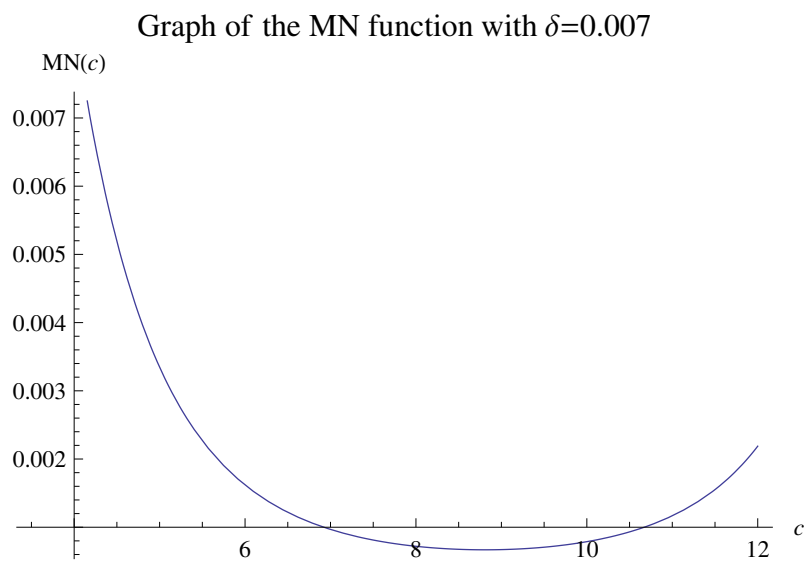

Figure 6: Here $n=4, \sigma=1$ and $b_{0}=1$. 
272 Boundary Elements and Other Mesh Reduction Methods XXXIII

\section{References}

[1] Cheng A.H.-D., Multiquadric and Its Shape Parameter-A Numerical Investigation of Error Estimate, Condition Number, and Round-Off Error by Arbitrary Precision Computation, submitted to Engineering Analysis with Boundary Elements.

[2] Wendland H., Multiscale Analysis in Sobolev Spaces on Bounded Domains, Numerische Mathematik 116, 493-517, 2010.

[3] Fleming W., Functions of Several Variables, Second Edition, Springer-Verlag, 1977.

[4] Luh L-T., The Shape Parameter in the Shifted Surface Spline, arXiv:1011.0124,2010.

[5] Luh L-T., The Shape Parameter in the Shifted Surface Spline II, in review.

[6] Luh L-T., A New Error Bound for Shifted Surface Spline Interpolation, Studies in Mathematical Sciences, Vol.1, No.1, 1-12, 2010. 